\section{AB0725 USING A REDUCED JOINT COUNT IN MEASURING DISEASE ACTIVITY IN PSORIATIC ARTHRITIS, IS IT LEGIT?}

M. Vis ${ }^{1}$, K. Wervers ${ }^{2}$, I. Tchetverikov ${ }^{3}$, A. Gerards ${ }^{4}$, M. Kok ${ }^{5}$, C. Appels ${ }^{6}$, L.-A. Korswagen ${ }^{7}$, M. Hazes ${ }^{1}$, J. Veris $^{8}$, H. van Groenendaal ${ }^{9}$, J. Luime $^{1}$ on behalf of DEPAR. ${ }^{1}$ Rheumatology; ${ }^{2}$ Erasmus MC; ${ }^{3}$ Rheumatology, Alber Schweitzer hospital; ${ }^{4}$ Vlietland ziekenhuis; ${ }^{5}$ Rheumatology, Maasstad ziekenhuis, Rotterdam; ${ }^{6}$ Rheumatology, Amphia ziekenhuis, Breda;

${ }^{7}$ Rheumatology, Sint Franciscus Gasthuis, Rotterdam; ${ }^{8}$ Rheumatology, RZWN, Goes; ${ }^{9}$ Rheumatology, RZWN, Roosendaal, Netherlands

Background: In Psoriatic arthritis (PsA) the pattern of joint involvement seems to be more heterogenic than Rheumatoid Arthritis (RA). Therefor the 66/68 joint count is frequently used in PsA scores. In this study we would like to investigate whether using less extensive joint counts influences PsA disease activity measures

Objectives: To evaluate the effect of using a 28,44 or $66 / 68$ joint count on Minimal Disease Activity (MDA).

Methods: Newly diagnosed PsA patients were included in the Dutch Early south-west Psoriatic Arthritis Registry (DEPAR) study between August 2013 and January 2017. Joint scores at baseline and 6 months were calculated using a 28 , 44 and 66/68 joint count. Consecutively MDA was calculated using each of these joint counts. MDA is defined as minimal disease activity in 5 out of 7 domains (swollen joints, tender joints, PASI, patient vas pain, patient vas global, HAQ, enthesitis)

Results: In total, 413 patients were included into the study, of which 320 had reached 6 months follow-up at the time of this abstract. Half of the patients were male $(49 \%)$, and mean age was 50 years (SD 13.8).

The percentage of patients with at least one involved joint (swollen or tender) at baseline decreased from $91 \%$ using the $66 / 68$ score to $88 \%$ with 44 joints and then to $80 \%$ with a 28 joint count (Table 1). At 6 month these scores were $66 \%$ $63 \%$ and finally $56 \%$ respectively for the 66/68, 44 and 28 joint count. After 6 months, 96 patients (30\%) had achieved MDA using the original 66/68 joint count. Using a 28 joint count, 10 more patients (10\% of the MDA population) were classified as having achieved MDA.

Table 1. Joint scores and MDA at baseline $(n=421)$ and 6 months $(n=320)$ using $66 / 68,44$ and 28 joint counts in a population of newly diagnosed PsA patients

\begin{tabular}{lccc}
\hline & \multicolumn{3}{c}{ Joints } \\
\hline T0 & $66 / 68$ & 44 & 28 \\
Swollen joint score, median & 2 & 1 & 1 \\
Tender joint score, median & 3 & 2 & 2 \\
Swollen joint score $>0, \mathrm{n}(\%)$ & $311(75)$ & $298(72)$ & $256(62)$ \\
Tender joint score $>0, \mathrm{n}(\%)$ & $346(84)$ & $331(80)$ & $290(70)$ \\
Involved joint $>0$ (swollen and/or tender), $\mathrm{n}(\%)$ & $375(91)$ & $363(88)$ & $329(80)$ \\
MDA, $\mathrm{n}(\%)$ & $56(14)$ & $65(16)$ & $70(17)$ \\
T6 & $66 / 68$ & 44 & 28 \\
Swollen joint score, median & 0 & 0 & 0 \\
Tender joint score, median & 1 & 1 & 0 \\
Swollen joint score $>0, \mathrm{n}(\%)$ & $125(41)$ & $117(38)$ & $100(32)$ \\
Tender joint score $>0, \mathrm{n}(\%)$ & $183(59)$ & $175(57)$ & $149(48)$ \\
Involved joint $>0$ (swollen and/or tender), $\mathrm{n}(\%)$ & $204(66)$ & $195(63)$ & $171(56)$ \\
MDA, $\mathrm{n}(\%)$ & $96(30)$ & $98(31)$ & $106(33)$ \\
\hline
\end{tabular}

Conclusions: Using reduced joint count joints in PsA misclassifies around $10 \%$ of patients as having no joint involvement. It also misclassifies about $10 \%$ of the MDA population of having achieved MDA while they have not. Full 66/68 joint counts remain recommended for measuring disease activity in PsA.

Disclosure of Interest: None declared

DOI: 10.1136/annrheumdis-2017-eular.6851

\section{AB0726 SUBCLINICAL ATHEROSCLEROSIS IN PATIENTS WITH ANKYLOSING SPONDYLITIS}

M. Ismail, A. Mosallam, M. Abdelaziz, A. Moshrif. Rheumatology, Faculty of Medicine, Al Azhar University, Assiut, Egypt

Background: Systemic inflammatory response of various autoimmune diseases has been reported as a cause of accelerated atherosclerosis which is a risk factor for cardiovascular complications.

Objectives: This study aimed to evaluate atherosclerosis in patients with ankylosing spondylitis (AS) and its relation to disease activity.

Methods: The study was carried out on thirty AS patients, who fulfilled the modified New York criteria. Thirty apparently healthy normal volunteers, age and sex matched served as control. Complete History taking, General and systemic examination, Body mass index (BMI), Bath AS disease activity index (BASDAl), Bath AS functional index (BASFI), lipid profile, inflammatory markers and serum glucose level were done. Based on BASADI score, patients were then classified to active and inactive groups comprised 11 patients $(37 \%)$ and 19 patients $(63 \%)$ respectively. Carotid intima-media thickness (CIMT) was measured using real-time gray-scale sonography. The intima-media thickness (IMT) of common carotid artery, carotid bulb and internal carotid artery was determined.

Results: 25 males $(83.3 \%)$ and 5 females $(16.7 \%)$ with mean age $39.1 \pm 12.38$, their mean disease duration $13.13 \pm 8.03$ while controls were 21 males $(70 \%)$ and 9 females $(30 \%)$ with mean age $39.7 \pm 12.6$.
While IMT was found significantly increased in patients $(0.87 \pm 0.49)$ versus controls $(0.5 \pm 0.12)(P<0.001)$, there was no significant difference regarding the presence of plaques and there was no stenosis found in cases or controls. No significant difference was found between active and inactive cases as regard to components of lipid profile. The mean IMT in active and inactive cases was $0.74 \pm 0.11$ and $0.72 \pm 0.17$ respectively with no significant difference. we found no significant correlation between IMT and different disease scores, ESR or CRP.

Conclusions: AS patients have a higher risk for developing atherosclerosis. IMT of common carotid artery could be added as a further investigation for detection and follow up of atherosclerosis in AS patients.

Disclosure of Interest: None declared

DOI: 10.1136/annrheumdis-2017-eular.4502

\section{AB0727 HIP OSTEOPOROSIS AND VERTEBRAL FRACTURES ARE ASSOCIATED WITH YOUNGER AGE AT DIAGNOSIS OF SPONDYLOARTHRITIS: RESULTS FROM THE COMOSPA STUDY}

M.H. Derakhshan ${ }^{1}$, N.J. Goodson ${ }^{2}$, J. Packham ${ }^{3}$, R. Sengupta ${ }^{4}$,

H. Marzo-Ortega ${ }^{5}$, A. Molto ${ }^{6}$, S. Siebert ${ }^{1}$ on behalf of BRITSpA and COMOSPA investigators. ${ }^{1}$ Institute of Infection, Immunity \& Inflammation, University of Glasgow, Glasgow; ${ }^{2}$ Academic Rheumatology Department, University of Liverpool, Liverpool; ${ }^{3}$ Haywood Rheumatology Centre, Keele University, Keele; ${ }^{4}$ Royal National Hospital for Rheumatic Diseases, Bath; ${ }^{5} \mathrm{NIHR}$, Leeds Teaching Hospitals Trust and LIRMM, University of Leeds, Leeds, United Kingdom; ${ }^{6}$ Paris Descartes University, Hôpital Cochin, Paris, France

Background: Spondyloarthritis (SpA) is associated with adverse bone health outcomes. It is not known whether age at disease diagnosis is associated with osteoporosis and fracture in later life.

Objectives: To examine the relationship between "younger age at SpA diagnosis" and risk of osteoporosis and fractures (vertebral and low-trauma peripheral).

Methods: COMOSPA is a large global cross-sectional study comprising 3984 patients with SpA from 23 countries1. We evaluated the association between "younger age at SpA diagnosis" (categorised into 5-year blocks) and vertebral fracture, low-trauma peripheral fracture, and osteoporosis $(\mathrm{t}<-2.5$ on bone densitometry) using logistic regression. Models were adjusted for age, sex, BMI, smoking, alcohol, NSAIDs, DMARDs, biologics, steroids and vitamin D level. Subgroup analysis was performed, stratified by peripheral and/or axial joint involvement.

Results: Data were available for 3923 participants (64\% male). Median age at SpA diagnosis was 33 (IQR 25-43) years. Younger age at SpA diagnosis was not associated with the risk of vertebral fractures in the entire cohort $(\mathrm{OR}=1.11$, $p=0.143$ ) nor in the subgroup with axial SpA involvement, but was increased in those with peripheral arthritis ( $\mathrm{OR}=1.26, p=0.014)$ indicating $26 \%$ excess risk for each 5 -year younger age at time of SpA diagnosis.

There was no significant association between younger age at diagnosis and the risk of low trauma peripheral fractures or spinal osteoporosis in the entire cohort or any subgroups. Younger age at SpA diagnosis was associated with higher risk of hip osteoporosis in the entire cohort ( $\mathrm{OR}=1.34, p=0.004)$ and both subgroups. Femoral neck osteoporosis demonstrated similar associations (Table).

Table 1. Association between "younger age at SpA diagnosis" and the risk of fractures and os teoporosis (multivariable)

\begin{tabular}{lccc}
\hline & OR & $95 \%$ Cl for OR & $p$ value \\
\hline Vertebral Fractures (All) & 1.11 & $0.96-1.29$ & 0.143 \\
Peripheral arthritis* subgroup & 1.26 & $1.05-1.51$ & 0.014 \\
Axial disease* subgroup $_{\text {Low trauma peripheral fracture (All) }}$ & 1.07 & $0.92-1.24$ & 0.370 \\
Spinal Osteoporosis (All) & 1.01 & $0.85-1.20$ & 0.924 \\
Peripheral arthritis* subgroup & 1.07 & $0.93-1.22$ & 0.362 \\
Axial disease* subgroup & 1.09 & $0.92-1.28$ & 0.338 \\
Hip Osteoporosis (All) & 1.04 & $0.90-1.20$ & 0.611 \\
Peripheral arthritis* subgroup & 1.34 & $1.10-1.64$ & 0.004 \\
Axial disease* subgroup & 1.30 & $1.02-1.64$ & 0.031 \\
Femoral neck Osteoporosis (All) & 1.41 & $1.14-1.75$ & 0.002 \\
\hline
\end{tabular}

${ }^{*}$ Subgroups overlap as many patients will have both axial and peripheral arthritis.

Conclusions: Younger age of $\mathrm{SpA}$ diagnosis was associated with higher risk of vertebral fracture in patients with peripheral arthritis but not axial disease in this study. Lack of association with vertebral fracture and spinal osteoporosis in axial disease may reflect difficulty in detecting spinal disease and osteoporosis in the presence of spinal pain and syndesmophytes. An association between hip osteoporosis and younger age at SpA diagnosis was noted.

References:

[1] Ann Rheum Dis 2016;75:1016-23.

Disclosure of Interest: None declared

DOI: 10.1136/annrheumdis-2017-eular.3439 